\title{
Is Stage 2 idiopathic osteonecrosis of the hip joint associated with version angles on imaging methods?
}

\author{
Cüneyd Günay, MD (D, Abdurrahman Özçelik, MD (1)
}

Department of Orthopedics and Traumatology, Eskişehir Osmangazi University, Faculty of Medicine, Eskişehir, Turkey

Femoral head osteonecrosis $(\mathrm{ON})$ has a progressive and destructive process, and is usually seen between the third and fifth decades of life. If not treated appropriately, be complete deterioration of the hip joint occurs in the future. Femoral head ON is more common than in the other parts of the human body, due to the greater loading on the joint in a standing position. The most common symptom is pain, particularly in the affected hip region, groin, buttock, and thigh. The most frequently affected parts of the hip are the superior section, known as the 10-12 o'clock position, and the most anterior part of the femoral head. ${ }^{[1,2]}$

The necrosis may result from trauma or may develop with no history of trauma. ${ }^{[3]}$ In non-traumatic cases, both hips are affected in about 40 to $70 \%$ of cases. ${ }^{[4]}$ In the literature, the known risk factors of $\mathrm{ON}$ have been reported to be alcoholism, smoking, systemic lupus erythematosus, human immunodeficiency virus infection,

Received: May 29, 2021

Accepted: September 24, 2021

Published online: November 19, 2021

Correspondence: Cüneyd Günay, MD. Eskişehir Osmangazi Üniversitesi Tıp Fakültesi Ortopedi ve Travmatoloji Anabilim Dalı, 26040 Meşelik, Eskişehir, Türkiye.

E-mail: cungunay@hotmail.com

Doi: $10.52312 /$ jdrs.2021.273

Citation: Günay C, Özçelik A. Is Stage 2 idiopathic osteonecrosis of the hip joint associated with version angles on imaging methods?. Jt Dis Relat Surg 2021;32(3):611-616.

(02021 All right reserved by the Turkish Joint Diseases Foundation

This is an open access article under the terms of the Creative Commons Attribution-NonCommercial License, which permits use, distribution and reproduction in any medium, provided the original work is properly cited and is not used for commercial purposes (http://creativecommons.org/licenses/by-nc/4.0/).

\section{ABSTRACT}

Objectives: In this study, we aimed to investigate whether anatomical variations of acetabulum were associated to idiopathic osteonecrosis $(\mathrm{ON})$ of the femoral head.

Patients and methods: Between January 2014 and March 2020, a total of 46 patients (32 males, 14 females; mean age: 43 years; range, 18 to 66 years) who were diagnosed with unilateral or bilateral $\mathrm{ON}$ of the hip joint and 44 healthy age- and sex-matched controls (30 males, 14 females; mean age: 46 years; range, 18 to 79 years) with no signs of $\mathrm{ON}$ of the hip joint were retrospectively analyzed. For both groups, measurements were taken of the anatomic parameters, including the acetabular version angle (VA), the sharp angle (SA), and the center-edge angle (CEA) on anteroposterior pelvic radiographs and magnetic resonance imaging (MRI) scans.

Results: The mean VA of both hips was found to be significantly smaller in Group 1 than in Group 2 on both MRI and X-ray $(14.9 \pm 4.1$ and $14.4 \pm 3.1$ vs. $17.3 \pm 3.9$ and $18.0 \pm 3.7$, respectively; $\mathrm{p}=0.004, \mathrm{p}<0.001)$. The mean SA of both hips was found to be significantly smaller in Group 1 than in Group 2 on both MRI and X-ray $(39.0 \pm 2.9$ and $38.9 \pm 2.8$ vs. $41.6 \pm 3.9$ and $40.8 \pm 4.9$, respectively; $p=0.001, p=0.036$ ). The mean CEA of both hips was found to be significantly larger in $\mathrm{ON}$ group than in control group on both MRI and X-ray $(36.7 \pm 6.1$ and $36.9 \pm 7.0 v s .32 .0 \pm 6.0$ and $31.5 \pm 7.5$, respectively; $\mathrm{p}<0.001, \mathrm{p}=0.001)$.

Conclusion: Version angles were found to be smaller in patients with $\mathrm{ON}$ and more acetabular coverage was observed. Greater coverage of the acetabulum may indicate early collapses of the femoral head even in Stage 2 ON patients. The smaller version angles may be associated with $\mathrm{ON}$.

Keywords: Acetabular coverage, acetabular version, femoral head osteonecrosis, idiopathic, magnetic resonance imaging, measurement, radiography.

chronic corticosteroid therapy, diabetes mellitus, hemoglobinopathies, myeloproliferative disorders (e.g., Gaucher disease, leukemia), coagulation disorders, pregnancy-related conditions, Caisson disease, chronic renal failure, and hyperlipidemia. ${ }^{[1,5]}$ However, it is not possible to identify a specific cause in some patients, and these cases are evaluated as 
idiopathic ON. The radiographic findings of $\mathrm{ON}$ vary according to the stage of the disease. ${ }^{[6]}$ Magnetic resonance imaging (MRI) is the gold-standard imaging method for diagnosis, detection, and planning the treatment strategy for patients with femoral head ON.

It is well-known that excessive acetabular anteversion and retroversion may lead to several abnormalities in the hip joint. ${ }^{[7]}$ Several studies have shown that acetabular anatomical abnormalities may increase intracapsular pressure due to the incomplete contact surface between the acetabulum and femoral head. ${ }^{[8-10]}$ However, there has not yet been full clarification of acetabular morphology variations. In the present study, we aimed to investigate whether there was any relationship between hip version and acetabular coverage in hip ON patients compared to healthy individuals. To the best of our knowledge, this is the first study in the literature to evaluate the anatomic parameters on MRI and X-rays in idiopathic $\mathrm{ON}$ of the femoral head (only Stage 2).

\section{PATIENTS AND METHODS}

This single-center, retrospective study was conducted at Eskişehir Osmangazi University, Faculty of Medicine, Department of Orthopedics and Traumatology between January 2014 and March 2020. A total of 46 patients (32 males, 14 females; mean age: 43 years; range, 18 to 66 years) who were diagnosed with unilateral or bilateral ON of the hip joint and 44 healthy age- and sex-matched controls (30 males, 14 females; mean age: 46 years; range, 18 to 79 years) with no signs of ON of the hip joint were included. Medical data of the patients and controls were retrieved from the hospital database. All patients were diagnosed from the clinical history, physical examination, radiography, and MRI (both radiology and clinical database reports). Group 1 consisted of 46 patients (79 hips [33 bilateral, 7 right, and 6 left]) with Stage 2 (Ficat and Arlet classification) ${ }^{[6]}$ femoral head ON, and Group 2 consisted of 44 healthy controls (88 hips) who underwent pelvis X-ray and MRI for a complaint of the hip joint pain and no findings of any hip pathology were detected. The etiology of all the ON patients in this study was idiopathic. Other possible risk factors for $\mathrm{ON}$, such as trauma, corticosteroid use, and hematological diseases were excluded from Group 1. Those having missing X-ray or MRI scans were also excluded. The pelvic radiography and MRI examinations of Group 2 patients were performed in the Department of Radiology of our institution, and they were selected consecutively from the orthopedic outpatient clinics. Group 2 subjects were excluded from the study, if the X-rays or MRI scans showed any hip pathology such as ON of the hip joint, hip fracture, hip labral lesion, hip dysplasia, femoroacetabular impingement, hip osteoarthritis, advanced stage coxarthrosis, malignancies of the hip joint such as bone tumor (with osteolytic and/or osteoblastic lesions), developmental disorders of the hip, prosthetic implants and/or internal fixation implants. Measurements were taken of the anatomic parameters of acetabular version angle (VA), the sharp angle (SA), and center-edge angle (CEA) on MRI and anteroposterior pelvic radiography of the patients and control group. All these angles were compared between the two groups and the measurements were compared on MRI and X-ray.

Both the pelvic MRI scans and X-rays were acquired with the patient lying supine. Using a digital radiographic system, the X-rays were taken from a tube to film distance of $100 \mathrm{~cm}$, and focus of the central beam on the pubic symphysis. All the angles assessed in this study were measured independently on both MRI and X-ray scans by two orthopedic surgeons with an experience of more than 15 years in this field. The contralateral uninvolved hip, and matched control patient hip was measured. The details of angle measurement techniques $^{[11]}$ are shown in Figures 1-4. For inter-observer reliability, two assessors, who were blinded to the measurement values of the other, measured each image separately.

A written informed consent was obtained from each participant. The study protocol was approved by the Eskişehir Osmangazi University, Faculty of Medicine Institutional Review Board (17.12.2019/20). The study was conducted in accordance with the principles of the Declaration of Helsinki.

\section{Statistical analysis}

Statistical analysis was performed using the IBM SPSS version 22.0 software (IBM Corp., Armonk, NY, USA). Descriptive data were expressed in mean \pm standard deviation (SD), median (min-max) or number and frequency, where applicable. The distributions of the VAs between Group 1 and Group 2 were found to be normal by using Shapiro-Wilk normality test. Sex distribution was analyzed using the chi-square test and age with the Student t-test. The intra-rater agreement was evaluated with intraclass correlation coefficient (ICC) with 95\% confidence interval (CI). All the measured angles were compared between the two groups with the Student t-test. The concordance of all the angles 


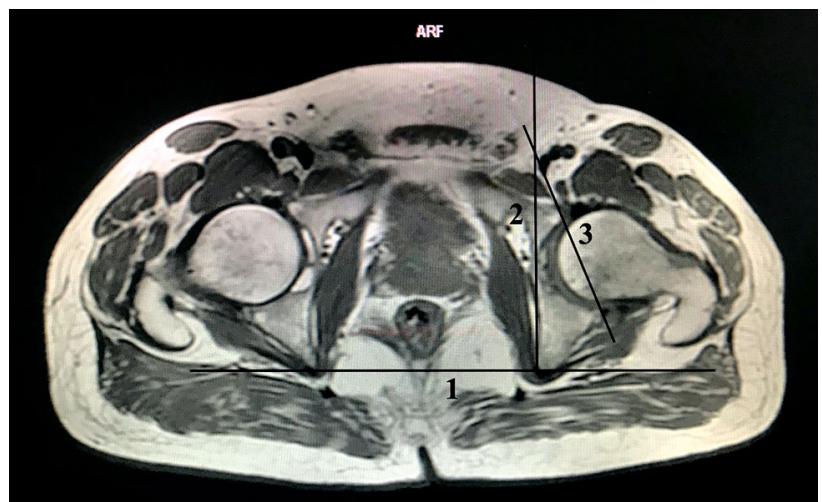

FIGURE 1. Axial MRI image (T1 image). Version angle on MRI is between Lines 2 and 3 .

Line 1: Inter-ischial line; Line 2: It is vertical to the Line 1; Line 3: It is connecting the anterior and posterior bony edges of the acetabular rim. MRI: Magnetic resonance imaging.

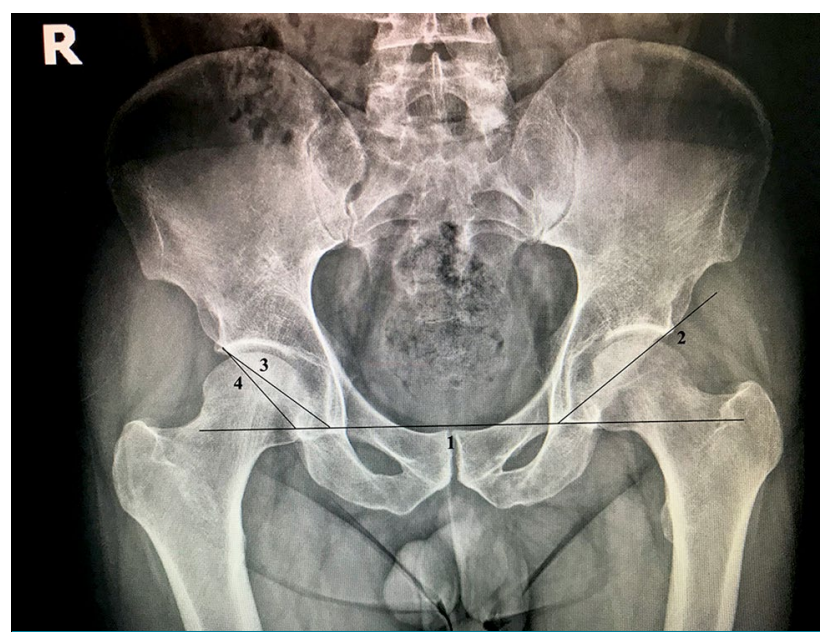

FIGURE 2. Anteroposterior pelvic radiography.

Line 1: Inter-ischial line; Line 2: It is drawn from the inferolateral part of the tear drop to the margin of the lateral acetabulum; Line 3: Same as Line 2; Line 4: It is drawn from the most lateral margin of the acetabulum to the most sclerotic part of the posterior lunate fossa of the acetabulum. Version angle (VA) on X-ray image: The angle is between Line 3 and Line 4. Sharp angle (SA): The angle is between Line 1 and Line 2.

was compared between MRI and X-ray using the Pearson correlation test. A $p$ value of $<0.05$ was considered statistically significant.

\section{RESULTS}

The angle measurements of the hips in both groups are shown in detail in Table I. The VA of hips both on MRI and X-ray was statistically significantly smaller in Group 1 than Group 2 ( $\mathrm{p}=0.004, \mathrm{p}<0.001$, respectively). The SA of hips both on MRI and X-ray was also statistically significantly smaller in the ON group than the control group $(\mathrm{p}=0.001, \mathrm{p}=0.036$, respectively). The CEA of the right-side hip on both

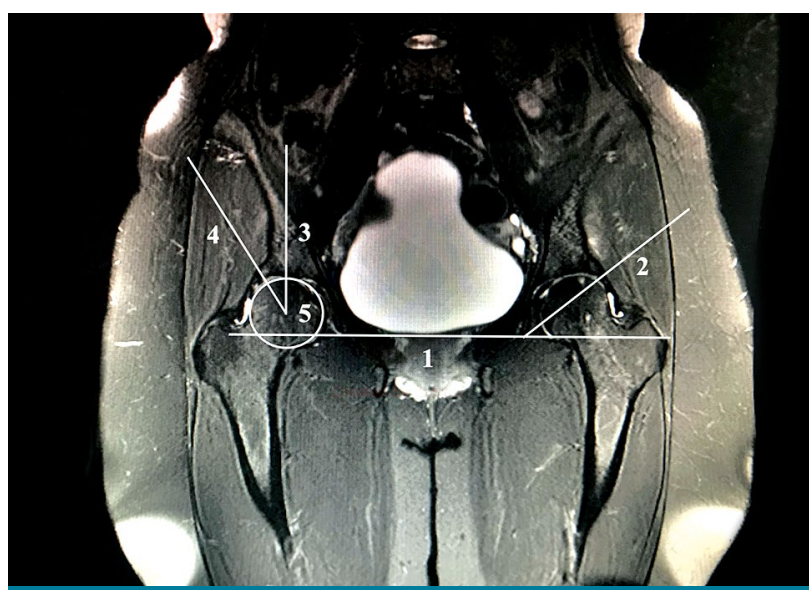

FIGURE 3. Magnetic resonance imaging coronal image (T1 image).

Line 1: Inter-ischial line; Line 2: It is drawn from the inferolateral part of the tear drop to the edge of the lateral acetabular sourcil; Line 3: It is vertical to the center of the femoral head; Line 4: It is drawn from the center of the femoral head to the lateral margin of the acetabular edge; Number 5: A circle which best fits on the femoral head. Sharp angle (SA) on magnetic resonance imaging (MRI): The angle between Lines 1 and 2. Center-edge angle (CEA) on MRI is formed between Lines 3 and 4 .

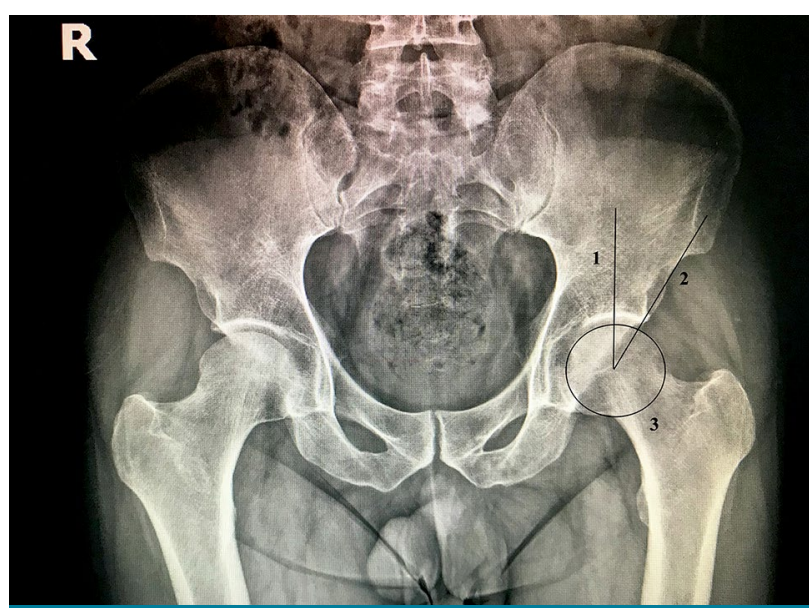

FIGURE 4. Anteroposterior pelvic radiography.

Line 1: It is vertical to the center of the femoral head; Line 2 is drawn from the center of the femoral head to the lateral margin of the acetabular rim; Number 3: A circle which best fits on the femoral head. Center-edge angle (CEA) on pelvic radiography: The angle between Lines 1 and 2 .

TABLE I

Acetabular version angle, sharp angle, and center-edge angle values of Group 1 and Group 2

\begin{tabular}{|lcccc|} 
& Group 1 & & Group 2 & \\
\cline { 2 - 2 } \cline { 5 - 5 } & Mean \pm SD & & Mean \pm SD & $p$ \\
\hline Hip MRI-VA & $14.9 \pm 4.1$ & & $17.3 \pm 3.9$ & $=0.004$ \\
Hip X-ray-VA & $14.4 \pm 3.1$ & & $18.0 \pm 3.7$ & $<0.001$ \\
Hip MRI-SA & $39.0 \pm 2.9$ & & $41.6 \pm 3.9$ & $=0.001$ \\
Hip X-ray-SA & $38.9 \pm 2.8$ & & $40.8 \pm 4.9$ & $=0.036$ \\
Hip MRI-CEA & $36.7 \pm 6.1$ & & $32.0 \pm 6.0$ & $<0.001$ \\
Hip X-ray-CEA & $36.9 \pm 7.0$ & & $31.5 \pm 7.5$ & $=0.001$ \\
\hline
\end{tabular}

SD: Standard deviation; MRI: Magnetic resonance imaging; VA: Version angle; SA: Sharp angle; CEA: Center-edge angle. 


\begin{tabular}{|c|c|c|c|c|c|c|}
\hline \multicolumn{7}{|c|}{$\begin{array}{c}\text { TABLE II } \\
\text { mographic data of the study population }\end{array}$} \\
\hline & \multicolumn{3}{|c|}{ Group $1(n=46)$} & \multicolumn{3}{|c|}{ Group $2(n=44)$} \\
\hline & $\mathrm{n}$ & $\%$ & Mean $\pm S D$ & $\mathrm{n}$ & $\%$ & Mean $\pm S D$ \\
\hline Age (year) & & & $43.4 \pm 11.8$ & & & $46.3 \pm 16.8$ \\
\hline \multicolumn{7}{|l|}{ Sex } \\
\hline Males & 32 & 69.6 & & 30 & 68.2 & \\
\hline Females & 14 & 30.4 & & 14 & 31.8 & \\
\hline Right hip & 7 & 15.2 & & - & - & \\
\hline Left hip & 6 & 13 & & - & - & \\
\hline Both hip & 33 & 71.7 & & 44 & 100 & \\
\hline
\end{tabular}

\begin{tabular}{|c|c|c|c|}
\hline \multicolumn{4}{|c|}{$\begin{array}{l}\text { TABLE III } \\
\text { The ICC values for acetabular version angle, sharp angle, } \\
\text { and center-edge angle on both MRI and X-ray }\end{array}$} \\
\hline & ICC & $95 \% \mathrm{Cl}$ & $p$ \\
\hline VA on MRI & 0.611 & $0.389-0.753$ & $<0.001$ \\
\hline VA on X-ray & 0.741 & 0.593-0.835 & $<0.001$ \\
\hline SA on MRI & 0.703 & $0.532-0.811$ & $<0.001$ \\
\hline SA on X-ray & 0.773 & $0.643-0.856$ & $<0.001$ \\
\hline CEA on MRI & 0.843 & $0.754-0.900$ & $<0.001$ \\
\hline CEA on X-ray & 0.891 & $0.829-0.931$ & $<0.001$ \\
\hline
\end{tabular}

MRI and X-ray was statistically significantly larger in the ON group than the control group $(\mathrm{p}<0.001$, $\mathrm{p}=0.001$, respectively).

Demographic details for the groups are presented in Table II. The differences in age and sex between the groups were not statistically significant $(\mathrm{p}=0.887$, $\mathrm{p}=0.336$, respectively).

The ICC was 0.691 (95\% CI: 0546-0806, $\mathrm{p}<0.001$ ) in Group 1 and 0.504 (95\% CI: 0.266-0.693, p<0.001) in Group 2 for all VA measurements between two assessors. The details of the ICC values for VA, SA, and CEA on both MRI and X-ray are presented in Table III. The MRI and radiography CEA, VA, and SA measurements were statistically significantly correlated $(r=0.852, p<0.001 ; r=0.646, p<0.001 ; r=0.726$, $\mathrm{p}<0.001$, respectively). A post-hoc power analysis was performed based on the given results in Table I for each VA both on MRI and X-ray. The power of this study was found to be $82 \%$.

\section{DISCUSSION}

Femoral head $\mathrm{ON}$ is an increasing health problem worldwide. The cause of $\mathrm{ON}$ is clearly identifiable in patients where bone vasculature, the bone, or the marrow are directly damaged..$^{[1,12]}$ However, the pathogenesis of $\mathrm{ON}$ remains a matter of debate in many patients, as there is still a lack of full understanding of the development mechanisms of this disorder. In addition to known identifiable causes of $\mathrm{ON}$, some cases $(30 \%)$ are defined as idiopathic, as the exact pathology cannot be determined. ${ }^{[13]}$ Acetabular VA has been blamed for many hip problems. ${ }^{[14]}$ The acetabulum anteversion angle may slightly increase throughout skeletal maturation. ${ }^{[14,15]}$ Reynolds et al. ${ }^{[16]}$ first described acetabular retroversion associated with a cross-over sign seen on pelvis radiography. Clinical problems may result from both increased retroversion and anteversion of the acetabulum. Excessive acetabular anteversion is a typical feature of acetabular dysplasia, and excessive retroversion is known to be associated with femoroacetabular impingement (pincer type), Perthes' disease, early coxarthrosis of the hip, and even slipped capital femoral epiphysis. Salter and periacetabular osteotomies may also result in iatrogenic retroversion. ${ }^{[15]}$ However, approximately one-third of the population suffering from this disease have no clear underlying etiology. It can be assumed that some structural anatomic acetabular shapes have a direct correlation with ON.

For several years, the quantification of acetabular version, computed tomography (CT) axial plane images are usually used. ${ }^{[7]}$ There has been acceptance of CT as the gold-standard method for the measurement of acetabular version. ${ }^{[7,17-19]}$ To avoid the exposure to radiation of CT scans, MRI has been 
increasingly used, not only for pediatric patients, but also for adults. For however, plain radiographs are often preferred owing to their cost-effectiveness, and they can be obtained more quickly and more easily than CT. There is also a high radiation risk for patients and higher costs to the healthcare system with the use of CT. A number of procedures have been described for the measurement of acetabular version on anteroposterior radiographs. ${ }^{[7,11]}$ In the current study, the measurements were made on MRI scans rather than the most commonly used method (CT). A recent study showed that zero echo time MRI could show similar angular measurement results compared to CT images, particularly in detecting the bony morphology of the hip joint. ${ }^{[17]}$ Therefore, to diagnose femoral head $\mathrm{ON}$ and for staging of the disease, all three angles were measured on MRI, and information was obtained about acetabular coverage of the femoral head. However, there cannot be a 100 percent guarantee about measurements on MRI made from either bony or soft tissue structures of the acetabulum. Muhamad et al. ${ }^{[15]}$ showed that acetabular version measurements on MRI either from the bony acetabulum or labrum were consistent with each other. Özçelik et al. ${ }^{[11]}$ also reported a statistically significant correlation between X-rays and CT measurements of acetabular version. In the current study, the three angles were consistent with each other on MRI and X-ray, and there was a statistically significant correlation between the two sets of measurements $(\mathrm{r}=0.852, \mathrm{p}<0.001)$.

Despite the importance of measuring acetabular version at the center of the femoral head, this does not fully describe the acetabular morphology. For instance, where there is pincer-type femoral acetabular impingement, acetabular retroversion may be limited to the superior aspect of the joint. Several studies have reported that acetabular anatomic problems which cause incomplete contact surface between the femoral head and the acetabulum may increase or alter intracapsular pressure of the hip joint. ${ }^{[8,9]}$ Osteonecrosis occurs in the anterolateral part of the femoral head. Therefore, it can be assumed that $\mathrm{ON}$ may be related to the increased intra-articular pressure, due to the femoral head coverage by both the anterior and lateral parts of the acetabulum. Thus, in this study, the three angles (VA, SA, CEA) were measured to obtain more information about the femoral head coverage. There are reports in the literature showing an association between less posterior acetabular coverage of the femoral head and VA with posterior hip dislocation in adolescent cases with a sports-related injury, even in the absence of a high-energy mechanisms. ${ }^{[20]}$
It was also reported that less acetabular coverage was found in idiopathic ON hips. In the aforementioned study, lower CEA, AHI (acetabular head index), acetabular depth ratio (ADR) and higher SA were found, compared to the control group. A study of patients from East Asia also showed that less acetabular coverage could be associated with the development of femoral head ON. ${ }^{[13]}$ In the current study, the CEA values were found to be higher and the SA values were lower in ON patients, compared to the control group, in contrast to the study by Zeng et al. ${ }^{[13]}$ Studies using CT and radiographs have suggested that malpositioning of the patient directly affects measurements of acetabular coverage of the femoral head. ${ }^{[11,21,22]}$ Dandachli et al. ${ }^{[23]}$ reported that every $5^{\circ}$ increase in pelvic anterior tilt resulted in a decrease of up to $5^{\circ}$ in acetabular anteversion. In the current study, special care was taken to avoid malpositioning of the patients while obtaining both pelvic X-rays and pelvic MRI. Taketa et al. ${ }^{[24]}$ reported that, in patients with developmental dysplasia of the hip with ON, the CEA did not accurately represent acetabular coverage. In contrast, the current study showed that the CEA values on both MRI and X-ray were higher in ON patients. This can be attributed to the collapse of the femoral head having started earlier than thought and some part of the femoral head having moved deep into the acetabular fossa, even in Stage 2 ON patients. Moreover, the lower SA value on both MRI and X-ray images showed greater acetabular coverage in $\mathrm{ON}$ patients, compared to the control group.

The ON group in this study only included Stage 2 patients, whereas Zeng et al. ${ }^{[13]}$ included both Stage 1 and Stage 2 patients. The difference in the results could be due to the lack of a standardization of the $\mathrm{ON}$ patients into one specific stage group. The current study results are inconsistent with a recent study by Zeng et al., ${ }^{[13]}$ as acetabular coverage in $\mathrm{ON}$ patients was found to be much greater than in the control individuals. A lower VA in the ON group in this study also indicates more acetabular coverage of the femoral head. At the end of this study, all the measured statistically significant angle values indicate early deterioration of the femoral head sphericity, and greater coverage of the femoral head was observed more in patients with ON.

Nonetheless, there were some limitations to this study. First, the study has a single-center, retrospective design with a relatively small sample size. Second, the angles were measured only once; therefore, the inter- and intra-observer reliability 
could have been less than, if the measurements had been taken twice.

In conclusion, this is the first study in the literature to compare acetabular anatomic angles on both MRI and X-ray images in idiopathic Stage 2 ON patients and healthy individuals. These small differences in the angle measurements in this study could be the reason for ON of the femoral head in patients with unknown etiology. The study results show more acetabular coverage in Stage $2 \mathrm{ON}$ patients than healthy individuals. Based on these findings, we can speculate that subchond ral collapse of the femoral head begins earlier than usually presumed, particularly in Stage 2 ON patients. Low VAs and greater coverage of the femoral head may be related to anterolateral ON. There is a need for further multi-center studies with larger series of ON patients to fully clarify these findings and the clinical relevance.

\section{Declaration of conflicting interests}

The authors declared no conflicts of interest with respect to the authorship and/or publication of this article.

\section{Funding}

The authors received no financial support for the research and/or authorship of this article.

\section{REFERENCES}

1. Aldridge JM 3rd, Urbaniak JR. Avascular necrosis of the femoral head: Etiology, pathophysiology, classification, and current treatment guidelines. Am J Orthop (Belle Mead NJ) 2004;33:327-32.

2. Moya-Angeler J, Gianakos AL, Villa JC, Ni A, Lane JM. Current concepts on osteonecrosis of the femoral head. World J Orthop 2015;6:590-601.

3. Şahin A, Agar A, Gülabi D, Ertürk C. Comparison of dynamic hip screw and antirotation screw with cannulated screw in the treatment of transcervical collum femoris fractures. Jt Dis Relat Surg 2020;31:320-7.

4. Carli A, Albers A, Séguin C, Harvey EJ. The medical and surgical treatment of ARCO stage-I and II osteonecrosis of the femoral head: A critical analysis review. JBJS Rev 2014;2:e2

5. Çalapkulu M, Kızılgül M, Sencar ME, Düğer H, Uçan B, Çakal E, et al. Avascular necrosis of the femoral head due to low-dose corticosteroid used in a patient with panhypopituitarism: A case report and literature review. Jt Dis Relat Surg 2020;31:390-4.

6. Ficat RP. Idiopathic bone necrosis of the femoral head. Early diagnosis and treatment. J Bone Joint Surg [Br] 1985;67:3-9.

7. Jamali AA, Mladenov K, Meyer DC, Martinez A, Beck M, Ganz R, et al. Anteroposterior pelvic radiographs to assess acetabular retroversion: High validity of the "cross-oversign". J Orthop Res 2007;25:758-65.

8. Hadley NA, Brown TD, Weinstein SL. The effects of contact pressure elevations and aseptic necrosis on the long- term outcome of congenital hip dislocation. J Orthop Res 1990;8:504-13.

9. Xie J, Naito M, Maeyama A. Intracapsular pressure and interleukin-1beta cytokine in hips with acetabular dysplasia. Acta Orthop 2010;81:189-92.

10. Wingstrand $H$. Intracapsular pressure in congenital dislocation of the hip. J Pediatr Orthop B 1997;6:245-7.

11. Özçelik A, Akalın $Y$, Çalışır C, İnan U, Ömeroğlu H. Definition of a quantitative measurement method for acetabular version in a plain radiograph in the healthy adult hip. Eklem Hastalik Cerrahisi 2015;26:2-5.

12. Mont MA, Hungerford DS. Non-traumatic avascular necrosis of the femoral head. J Bone Joint Surg Am 1995;77:459-74.

13. Zeng J, Zeng Y, Wu Y, Liu Y, Xie H, Shen B. Acetabular anatomical parameters in patients with idiopathic osteonecrosis of the femoral head. J Arthroplasty 2020;35:331-4.

14. Maruyama M, Feinberg JR, Capello WN, D'Antonio JA. The Frank Stinchfield Award: Morphologic features of the acetabulum and femur: Anteversion angle and implant positioning. Clin Orthop Relat Res 2001;(393):52-65.

15. Muhamad AR, Freitas JM, Bomar JD, Hosalkar H. Acetabular version on magnetic resonance imaging: Analysis of two different measuring techniques. Hip Int 2012;22:672-6.

16. Reynolds D, Lucas J, Klaue K. Retroversion of the acetabulum. A cause of hip pain. J Bone Joint Surg $\mathrm{Br}$ 1999;81:281-8.

17. Breighner RE, Bogner EA, Lee SC, Koff MF, Potter HG. Evaluation of osseous morphology of the hip using zero echo time magnetic resonance imaging. Am J Sports Med 2019;47:3460-8.

18. Perreira AC, Hunter JC, Laird T, Jamali AA. Multilevel measurement of acetabular version using 3-D CT-generated models: Implications for hip preservation surgery. Clin Orthop Relat Res 2011;469:552-61.

19. Werner CM, Copeland CE, Ruckstuhl T, Stromberg J, Turen $\mathrm{CH}$, Kalberer F, et al. Radiographic markers of acetabular retroversion: Correlation of the cross-over sign, ischial spine sign and posterior wall sign. Acta Orthop Belg 2010;76:166-73.

20. Novais EN, Ferrer MG, Williams KA, Bixby SD. Acetabular retroversion and decreased posterior coverage are associated with sports-related posterior hip dislocation in adolescents. Clin Orthop Relat Res 2019;477:1101-8.

21. van Bosse HJ, Lee D, Henderson ER, Sala DA, Feldman DS. Pelvic positioning creates error in CT acetabular measurements. Clin Orthop Relat Res 2011;469:1683-91.

22. Richards PJ, Pattison JM, Belcher J, Decann RW, Anderson S, Wynn-Jones C. A new tilt on pelvic radiographs: A pilot study. Skeletal Radiol 2009;38:113-22.

23. Dandachli W, Ul Islam S, Richards R, Hall-Craggs M, Witt J. The influence of pelvic tilt on acetabular orientation and cover: A three-dimensional computerised tomography analysis. Hip Int 2013;23:87-92.

24. Taketa M, Fujii T, Kubota H, Nakashima Y, Noguchi Y, Iwamoto Y. Correlation between center-edge angle and acetabulum-head index in developmental dysplasia of the hip with avascular necrosis of the femoral head. J Pediatr Orthop B 2003;12:215-8. 